\title{
Biological Atomic Force Microscopy for Imaging Gold-Labeled Liposomes on Human Coronary Artery Endothelial Cells
}

\author{
Ana-María Zaske, ${ }^{1}$ Delia Danila, ${ }^{1}$ Michael C. Queen, \\ Eva Golunski, ${ }^{2}$ and Jodie L. Conyers ${ }^{3}$ \\ ${ }^{1}$ Division of Cardiology, Department of Internal Medicine, The University of Texas Health Science Center at Houston, 1881 East Road, \\ Houston, TX 77054, USA \\ ${ }^{2}$ Division of Cardiology, Department of Internal Medicine, The University of Texas Health Science Center at Houston, 1841 East Road, \\ Houston, TX 77054, USA \\ ${ }^{3}$ Center of Translational Injury Research, The University of Texas Health Science Center at Houston, 6410 Fannin Street, Houston, \\ TX 77030, USA
}

Correspondence should be addressed to Ana-María Zaske; ana.m.zaske@uth.tmc.edu

Received 28 November 2012; Accepted 18 January 2013

Academic Editor: Venkata Vamsi Krishna Venuganti

Copyright ( 2013 Ana-María Zaske et al. This is an open access article distributed under the Creative Commons Attribution License, which permits unrestricted use, distribution, and reproduction in any medium, provided the original work is properly cited.

\begin{abstract}
Although atomic force microscopy (AFM) has been used extensively to characterize cell membrane structure and cellular processes such as endocytosis and exocytosis, the corrugated surface of the cell membrane hinders the visualization of extracellular entities, such as liposomes, that may interact with the cell. To overcome this barrier, we used $90 \mathrm{~nm}$ nanogold particles to label FITC liposomes and monitor their endocytosis on human coronary artery endothelial cells (HCAECs) in vitro. We were able to study the internalization process of gold-coupled liposomes on endothelial cells, by using AFM. We found that the gold-liposomes attached to the HCAEC cell membrane during the first 15-30 min of incubation, liposome cell internalization occurred from 30 to 60 min, and most of the gold-labeled liposomes had invaginated after $2 \mathrm{hr}$ of incubation. Liposomal uptake took place most commonly at the periphery of the nuclear zone. Dynasore monohydrate, an inhibitor of endocytosis, obstructed the internalization of the gold-liposomes. This study showed the versatility of the AFM technique, combined with fluorescent microscopy, for investigating liposome uptake by endothelial cells. The $90 \mathrm{~nm}$ colloidal gold nanoparticles proved to be a noninvasive contrast agent that efficiently improves AFM imaging during the investigation of biological nanoprocesses.
\end{abstract}

\section{Introduction}

The development of biologically active nanoparticles that can facilitate the delivery of therapeutic and/or diagnostic agents to precise regions within the human body has gained widespread attention in the past 10 years. While many have successfully designed and tested such nanovectors in vivo, little is known about how these particles dynamically interact with cell membranes. Atomic force microscopy (AFM), a surface analytical technique that generates nanoscale topographical images under physiological-like conditions [1], combined with fluorescence microscopy is an attractive tool for characterizing nanoparticle-cell membrane interactions and may afford the opportunity to image biological processes, such as cellular endocytosis of nanovectors and reorganization of the cytoskeleton, in real time.

Liposomes were one of the first classes of engineered nanoparticles to be used to deliver drugs within the human body. One of the major disadvantages of using standard liposome formulations is their rapid clearance from circulation due to uptake by the reticuloendothelial system. To resolve this problem, long-circulating liposomes were developed in the early 1990s [2, 3]. Long-circulating liposomes have polyethylene glycol (PEG) derivatives attached to their surfaces. The conformational flexibility of PEG chains creates a sterical barrier that allows the liposomes to evade uptake by the reticuloendothelial system and remain in circulation for a longer time, increasing the possibility 
for targeting [4]. The ability to pegylate liposomes, for the purpose of mitigating a systemic immune response and/or enhancing prolonged vascular circulation, has made these particles attractive nanovectors for increasing the efficacy of therapeutics. For instance, Doxil entraps doxorubicin (an anthracycline antibiotic used as a chemotherapy agent) within a pegylated liposome for treating Kaposi's sarcoma [5].

Our laboratory is designing liposomes that are immunospecific to inflamed endothelium, a hallmark of atherosclerosis, for the purpose of imaging lipid-rich plaques before the onset of clinical symptoms (e.g., angina, coronary stenosis, etc.). While in vivo studies of liposomes plaque specificity (imaged by computed tomography) are currently underway, we are also determining how these liposomes interact with endothelial cells that have been induced to express an inflammatory response.

Structure recognition can be a challenge during AFM imaging. Some nanoparticles can also be used as imaging contrast agents and as reinforcement elements to improve visual enhancement [6]. Colloidal gold has excellent detection capabilities for single-molecule tracking $[7,8]$. The aim of this project was to standardize the use of gold nanoparticles as a nontoxic means to detect pegylated liposomes by AFM, on the membranes of live human coronary artery endothelial cells (HCAECs), to elucidate internalization processes.

\section{Experimental Methods}

2.1. Materials. 1,2-Dipalmitoyl-sn-glycero-3-phosphocholine (DPPC); cholesterol; 1,2-distearoyl-sn-glycero-3-phosphoethanolamine-N-[carboxy (polyethyleneglycol) 2000] (DSPE-PEG (2000)-COOH); and 1,2-distearoyl-sn-glycero3-phosphoethanolamine-N-[methoxy (polyethyleneglycol)2000] (DSPE-PEG (2000)) were purchased from Avanti Polar Lipids (Alabaster, AL, USA). N-(Fluorescein-5-thiocarbambamoyl)-1,2-dihexa-decanoyl-sn-glycero-3-phosphoethanolamine (DHPE-FITC) was purchased from Molecular Probes (Eugene, OR, USA). Dimethyl sulfoxide anhydrous $99.9 \%$ (DMSO), N-(3-dimethylaminopropyl)-N' -ethylcarbodiimide hydrochloride (EDC), chloroform A.C.S. reagent $99.8 \%$ with amylenes as stabilizer, 2-(N-morpholino) ethanesulfonic acid (MES), collagen from calf skin, neutralbuffered formalin $10 \%$, and dynasore monohydrate were purchased from Sigma-Aldrich (St. Louis, MO, USA). N-Hydroxysulfosuccinimide (sulfo-NHS) was purchased from Pierce (Rockford, IL, USA). Amine-PEG conjugated spherical gold nanoparticles (90-nm gold nanoparticles) were purchased from Nanopartz (Salt Lake City, UT, USA). HCAECs (CC-2585 Lot \# EN000307), endothelial cell basal medium-2 (EBM-2), and the endothelial cell growth medium (EGM-2) BulletKit (SingleQuots) were purchased from Lonza (Walkersville, MD, USA). Eight-well Lab-Tek Chamber Permanox Slides were purchased from Nalge Nunc Int. (Rochester, NY, USA). Ruby red mica sheets $\left(1 \times 3^{\prime \prime}\right)$, for liposome AFM imaging, were purchased from Electron Microscopy Sciences (Hatfield, PA, USA). AFM cantilevers for tapping mode (RTESP, $f_{0}=262-325 \mathrm{kHz}, k=20-80 \mathrm{~N} / \mathrm{m}$ ) and contact mode (DNP-S, fo $=12-24 \mathrm{kHz}, k=0.06 \mathrm{~N} / \mathrm{m}$ ) were purchased from Bruker Corporation (formerly Veeco Metrology) (Santa Barbara, CA, USA).

2.2. Preparation of Long-Circulating Liposomes. We synthesized long-circulating liposomes by using DPPC and our previously published procedure [9]. The liposomes were composed of DPPC and cholesterol in a 3:1 molar ratio. The linker lipid DSPE-PEG (2000)-COOH was incorporated at a molar ratio of $3: 1: 0.3$, DPPC: cholesterol: linker. The synthesized liposomes were sterically stabilized by addition of a $1 \%$ solution of DSPE-PEG (2000) in chloroform. For fluorescence microscopy experiments, $2 \mathrm{~mol} \%$ of fluorescently labeled DHPE-FITC was incorporated into the lipid mixture. The liposomes were prepared by hydration of the dry lipid film as described by others [10, 11]. Briefly, the lipids were dissolved in chloroform, which was then carefully evaporated with a rotary evaporator (Büchi Corporation). The resulting dry lipid film was hydrated at $65^{\circ} \mathrm{C}$ with MES (100 mM, pH 5.5) to a final phospholipid concentration of $30 \mathrm{mM}$. Vigorous shaking of the solution produced large multilamellar vesicles of various sizes. Using an extruder (Avanti Polar Lipids), we passed the multilamellar vesicle solution 21 times through $0.2 \mu \mathrm{m}$ polycarbonate membranes at $65^{\circ} \mathrm{C}$ to yield a homogeneous solution of unilamellar vesicles with an average diameter of $129 \mathrm{~nm}$, as determined by dynamic light scattering (DLS) measurements.

2.3. Dynamic Light Scattering Measurements. DLS is one of the most common techniques to determine the radius of spherical particles in Brownian motion in a solution [12]. The size distribution of the liposomes was measured with a DLS Malvern Nano-ZS Zetasizer (Malvern Instruments Ltd.; Malvern, Worcestershire, UK). The Zetasizer was loaded with a liposome solution ratio $1: 200(70 \mu \mathrm{M})$ in $1 \mathrm{X}$ phosphatebuffered saline (PBS, $\mathrm{pH} 7.4$ ) solution and ran 13 cycles to obtain a measurement. This instrument is also used to obtain the zeta potential charge. DPPC liposomes have a resultant negative potential of $-11.4 \mathrm{mV}$, indicating that the liposomes are stable and that they resist aggregation. Data were analyzed using the Dispersion Technology Software version 4.20 (Malvern Instruments Ltd.).

2.4. Preparation of Long-Circulating Gold-Liposomes. Conjugated spherical gold nanoparticles with a diameter of $90 \mathrm{~nm}$ (Nanopartz) were covalently linked to the long-circulating liposomes for AFM detection. "Gold-liposomes" were prepared by attaching the gold nanoparticles at the distal terminals of DSPE-PEG (2000)-COOH linker lipids, which have free carboxylic groups available for activation [9, 13]. After the dry lipid hydration and liposome isolation procedures described above, $300 \mu \mathrm{L}$ of the $30 \mathrm{mM}$ liposome solution was incubated with the activating reagents EDC $(9 \mathrm{mg})$ and sulfo-NHS (11 mg) for $4 \mathrm{hrs}$ at room temperature. The free activating reagents were then removed by dialysis through a 6,000-8,000 MW cut-off membrane against PBS ( $\mathrm{pH} 7.4$ ). Then, $100 \mu \mathrm{L}$ of gold nanoparticles was added to $400 \mu \mathrm{L}$ of the activated liposome solution and incubated overnight at room temperature. The unbounded gold nanoparticles were removed by dialysis through a 500,000 MW cut-off 
membrane against PBS ( $\mathrm{pH}$ 7.4), and the average size of the "gold-liposomes" was measured by DLS.

\subsection{Atomic Force Microscopy}

2.5.1. Liposome Imaging. The liposomes were deposited on mica for AFM analysis by using a modification of the procedure of Ramachandran et al. [14]. Ruby red mica circles $\left(11 \mathrm{~mm}\right.$ diameter) were first glued to a $3^{\prime \prime} \times 1^{\prime \prime}$ glass slide with Scotch super glue gel. Both uncoupled liposomes and gold-coupled liposomes were left to stand on freshly cleaved mica during $10 \mathrm{~min}$. The liposomes were then fixed with $10 \%$ neutral buffered formalin for $10 \mathrm{~min}$, washed 3 times with ultrapure water (Barnstead water system, Thermo Scientific; Dubuque, IA, USA), and dried in a sterilGARD III hood flow before scanning. AFM liposome imaging was performed in tapping mode in air with RTESP cantilevers $(f o=262-$ $325 \mathrm{kHz}, k=20-80 \mathrm{~N} / \mathrm{m})$.

2.5.2. Cell Imaging. For the endocytosis studies, $2 \times 10^{4}$ cells/ well of HCAECs were seeded in a collagen- $(50 \mu \mathrm{g} / \mathrm{mL})$ coated 8-well slide system, with EBM-2 supplemented with an EGM2 Bulletkit (SingleQuots) to obtain $80 \%$ confluence after $24 \mathrm{hr}$ of incubation at $37^{\circ} \mathrm{C}$ in a $5 \% \mathrm{CO}_{2}$ atmosphere. The cells were then treated with the gold-labeled liposomes at a final concentration of $1.525 \mathrm{mM}$ (solution ratio $1: 10$ in culture medium) for four different incubation times $(15,30,60$, and $120 \mathrm{~min}$ ) at $37^{\circ} \mathrm{C}$. During the incubations, the slide chambers were agitated at $30 \mathrm{rpm}$ with an Orbit P4 Digital Shaker (Labnet, Edison, NJ, USA). The cells were then washed 3 times with culture medium to remove unbounded liposomes and fixed for $15 \mathrm{~min}$ with $10 \%$ neutral buffered formalin.

AFM studies were performed on "never-dried" fixed cells, after the four incubation periods, to investigate liposomemembrane interactions. Liquid scanning was performed in contact mode with DNP-S cantilevers $\left(f_{o}=12-24 \mathrm{kHz}\right.$, $k=0.06 \mathrm{~N} / \mathrm{m}$ ). AFM was performed with a BioScope II Controller (Bruker Corporation). Image analysis was conducted with the Research NanoScope software version 7.30. For routine liposome detection, we used a Nikon TE2000-E inverted optical fluorescence microscope (Nikon Instruments, Inc. Lewisville, TX, USA) integrated to the bioscope system. Epifluorescence images were taken using an FITC-HYQ filter set (excitation 460-500 wavelengths) with 20 and $40 \mathrm{X}$ objectives, respectively. The cells that emitted a positive fluorescent signal by the FITC-labeled liposomes were selected for AFM scanning. The results from the liposome AFM measurements were also verified by DLS analysis.

2.6. Inhibition of Endocytosis. The effect of dynasore monohydrate on cellular uptake of liposomes was also investigated. Dynasore monohydrate is a GTPase inhibitor that targets dynamin and blocks endocytosis $[15,16]$. Dynamin has an essential role of vesicle formation in receptor-mediated endocytosis $[17,18]$. The dynasore monohydrate was initially diluted to $20 \mathrm{mM}$ in DMSO (99.9\%) and stored in $20-\mu \mathrm{L}$ aliquots at $-20^{\circ} \mathrm{C}$. At the time of the experiments, it was diluted to $80 \mu \mathrm{M}(0.4 \% \mathrm{DMSO})$ in EBM-2 that contained no serum or albumin [19]. The HCAECs were then treated with dynasore monohydrate $(80 \mu \mathrm{M})$ for $15 \mathrm{~min}$ and agitated at $30 \mathrm{prm}$ at $37^{\circ} \mathrm{C}$ before being incubated for $60 \mathrm{~min}$ with gold-labeled liposomes diluted 1:10 (1.525 mM) in EBM-2 supplemented with the EGM-2 BulletKit. We chose to investigate dynasore monohydrate's blocking effect after $60 \mathrm{~min}$ of incubation because, as described later, we learned that the "gold liposomes" had been taken up by the cells at that point in time. This is also in agreement with the work of Mastrobattista et al. [20], who reported that the $60 \%$ of cellbound immunoliposomes are taken up by bronchial epithelial cells within $1 \mathrm{~h}$ of incubation.

\section{Results and Discussion}

Our initial attempts to monitor the endocytosis of FITC liposomes by HCAECs required the utilization of an imaging contrast agent to improve visual enhancement during AFM scanning. FITC-labeled liposomes were not detectable during AFM imaging when incubated with HCAECs (see Figure S1 in Supplementry Materials avalible online at http://dx.doi .org/10.1155/2013/875906). Positive fluorescence signaling was detected from the fluorescently labeled lipid, but uncoupled liposomes were not detectable on the surface of the cell membrane during AFM scanning. This difficulty led us to use colloidal gold to perform single-molecule tracking, a common technique practiced in morphological studies $[7,8]$. For this reason, $90 \mathrm{~nm}$ gold particles were coupled to FITClabeled liposomes to facilitate particle identification in AFM profiles.

\subsection{Characterization of Uncoupled Liposomes and Gold-Coupled Liposomes}

3.1.1. DLS Measurements. The average diameter of the nongold-coupled liposomes, as determined by DLS, was $129 \pm$ $1.3 \mathrm{~nm}$ (Figure 1(a)), and a polydispersity index (PDI) of 0.126 . The DLS results represent the averages of three different measurements (13 runs each) per sample. For the goldcoupled liposomes (Figure 1(b)), the DLS results showed a major peak at $285 \pm 5.3 \mathrm{~nm}$. The PDI for the gold-liposomes was 0.689 .

The polydispersity index indicates the variation in particle size. Its denomination can vary from 0 to 1 . A value of 1 means that the sample is very polydisperse and a value of 0 means that the particle size does not vary. Values below 0.2 indicate that a sample is monodisperse [21, 22]. Therefore, the PDI values reported here indicate that the noncoupled liposomes were very monodisperse. However, the goldcoupled liposomes had a more heterogeneous formulation, some containing only one or two gold particles and some having many (clusters).

3.1.2. AFM Analysis of Liposomes. The structural properties of uncoupled and gold-coupled liposomes were analyzed using AFM. Both types of liposomes were imaged after fixation with $10 \%$ formalin to ensure the preservation of their original structure. AFM was performed in tapping mode in air with RTESP cantilevers. Figure 2 shows a typical AFM image of non-gold-coupled liposomes deposited on fresh 


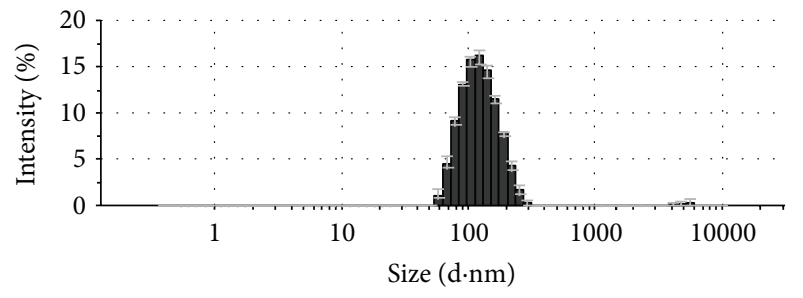

(a)

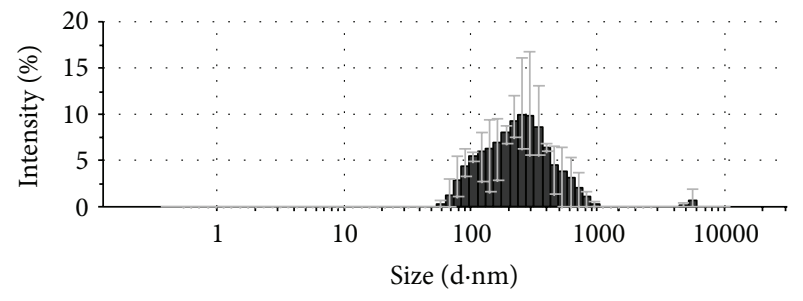

(b)

FIGURE 1: The hydrodynamic radii of noncoupled (a) and gold-coupled liposomes (b), estimated by DLS. Uncoupled liposomes (a) observed a mean diameter value of $129 \pm 1.3 \mathrm{~nm}$. The size distribution for gold-labeled liposomes (b) described a major peak for particles about $285 \pm 5.3 \mathrm{~nm}$ in size. DLS results represent the averages of 13 runs at three different measurements per sample.

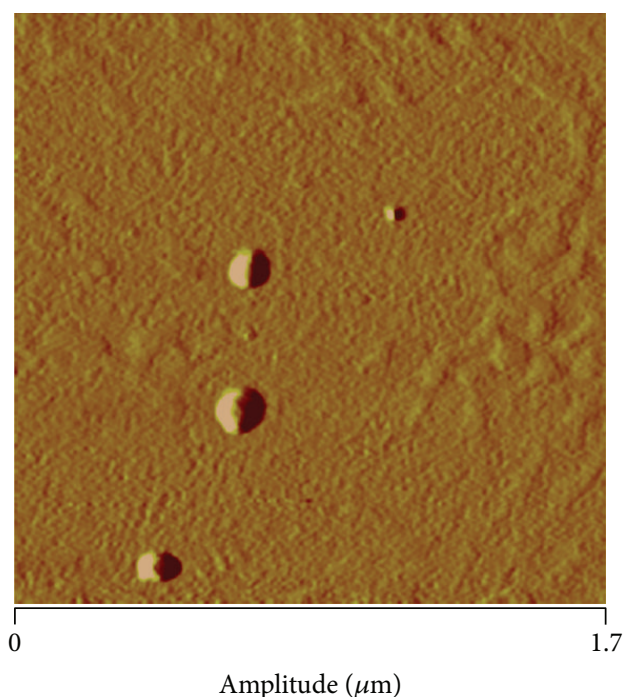

(a)

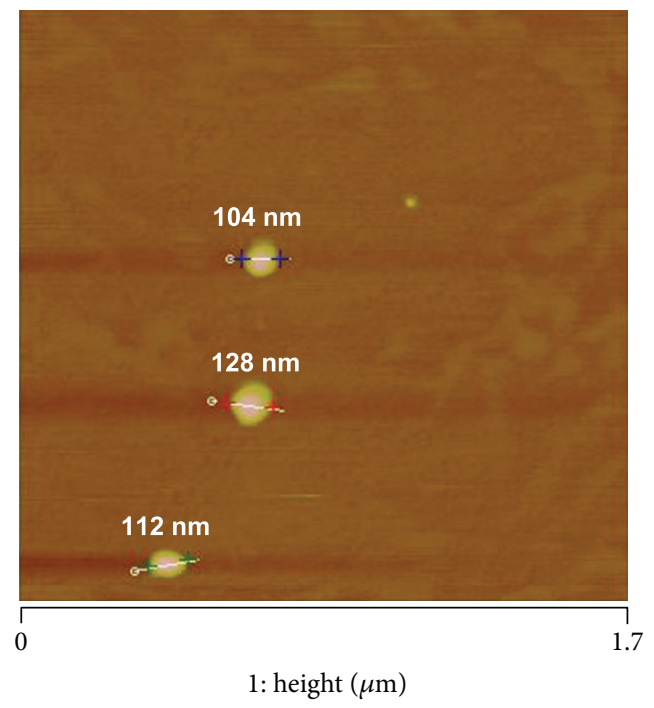

(b)

Figure 2: AFM images of non-gold-coupled liposomes visualized in amplitude (a) and height (b) modes to $1.7 \mu \mathrm{m}(x-y)$. The AFM section analysis of these liposomes showed an average diameter of $121.5 \pm 27 \mathrm{~nm}$ when deposited on mica and scanned in air. This result was comparable to that of the DLS analysis $(129 \pm 1.3 \mathrm{~nm})$. Scan obtained in tapping mode using RTESP tips $(f o=262-325 \mathrm{kHz}, k=20-80 \mathrm{~N} / \mathrm{m})$.

cleaved mica and fixed with formalin. AFM analysis of these liposomes showed an average diameter of $121.5 \pm 27 \mathrm{~nm}$. This result was comparable to the diameter measurement obtained by DLS $(129 \pm 1.3 \mathrm{~nm})$.

To enhance AFM imaging, nanogold particles were covalently linked to FITC liposomes. We choose $90 \mathrm{~nm}$ gold particles to facilitate visualization within the cell membrane taking into consideration that $\sim 120 \mathrm{~nm}$ gold nanoparticles are normally used for thermal ablation. Figure 3 shows AFM imaging of the geometric structure of a typical gold-liposome cluster (303 nm in size) consisting of three distinctive particles with individual diameters of 95,86 , and $80 \mathrm{~nm}$.

According to DLS measurements, these gold-liposome complexes had an average size of $285 \pm 5.3 \mathrm{~nm}$ (3 different measurements with 13 runs each). The arrangement and geometrical structure of the gold-liposome clusters changed, but the average size remained similar in different formulations $(n=5)$.

3.2. Endocytosis and Liposome Coupling. The surface topology and characteristics of biological membranes can routinely be described using biological AFM instruments
$[23,24]$. Nevertheless, the application of this technique has rarely been approached to resolve kinetics for liposome cell uptake. To investigate how endocytosis takes place within endothelial cells, we used $90 \mathrm{~nm}$ gold particles to track FITClabeled liposomes. Figure 4 shows sequential AFM images illustrating how the "gold-liposomes" internalize the plasma membrane.

After 15 min of incubation (Figures 4(a) and 4(b)), some of the coupled liposomes were already attached to the cells. They still quivered when probed with the AFM tip during scanning (seen as liposome drifting in Figure 4(a)). Nevertheless, the "gold-liposomes" were strongly bonded to the cell membrane at this time, given that the liposomes remained attached to the cell when probed. At $30 \mathrm{~min}$, the liposome clusters started to enter the cells (not shown). Actual endocytosis was seen in samples incubated for $60 \mathrm{~min}$ (Figures 4(c) and 4(d)). Figures 4(c) and 4(d) clearly show how the plasma membrane is enclosing the extracellular material and gradually engulfing it (see also Figure 5(a), at a smaller scan area). The corresponding fluorescence images in Figures 4(d) and 5(b) show strong positive signals of the two "gold-liposome" clusters (indicated by arrows) that were 


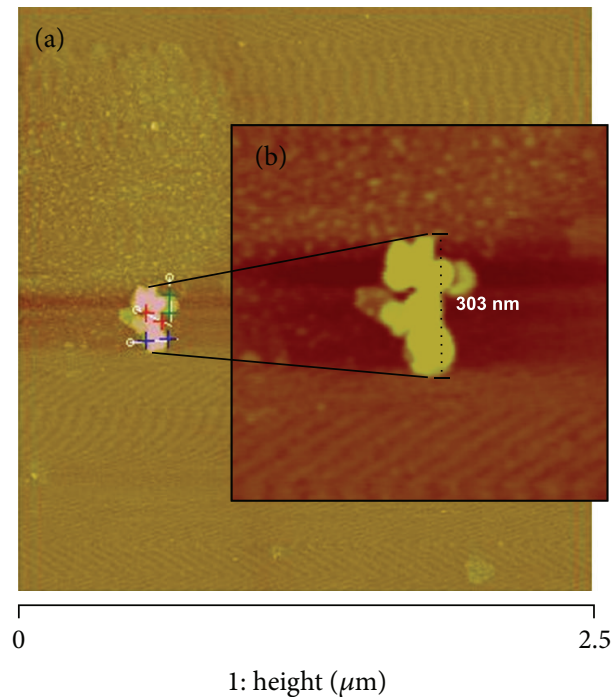

FIGURE 3: AFM images of FITC-labeled liposomes coupled to $90 \mathrm{~nm}$ gold particles and scanned on fresh cleaved mica. (a) Height image with section analysis measuring the diameter of three particles $(95,86$, and $80 \mathrm{~nm}$, resp.) that made up a typical gold-liposome cluster. (b) Additional digital zoom to $1 \mu \mathrm{m}^{2}$ taken from the original scan at $2.5 \mu \mathrm{m}(x-y)$. The DLS analysis showed that the gold-liposome complexes had a diameter of $285 \pm 5.3 \mathrm{~nm}$. Tapping mode in air using RTESP cantilevers $\left(f_{o}=262-325 \mathrm{kHz}, k=20-80 \mathrm{~N} / \mathrm{m}\right)$.

selected for AFM scanning. One of these clusters was about $3.2 \mu \mathrm{m}$ and the other was $3.8 \mu \mathrm{m}$ in diameter. Liposomes incubated with HCAECs for 120 min were almost completely internalized (Figure 4(e)).

These studies demonstrated that using colloidal gold nanoparticles as an image-enhancement tool did not hinder endocytosis and that uptake of the liposomes took place in $120 \mathrm{~min}$. The time course of the endocytosis can be summarized as follows: liposome attachment to the plasma membrane (15-30 min), internalization (30-60 min), and liposome uptake completed $(120 \mathrm{~min})$. These results agree with the findings reported by Ramachandran et al. [14], who studied endocytosis of cisplatin-encapsulated liposomes. In that study, the cisplatin produced liposomes significantly stiffer than nonencapsulated liposomes, which facilitated their detection by AFM scanning.

On the other hand, our negative controls (HCAEC incubated with "gold-liposomes" for 60 and $120 \mathrm{~min}$, which did not show signals detectable by fluorescence imaging) had smooth and even membrane surfaces (Figures 5(c) and 5(d)). Signs of elevated or raised areas were not present during AFM scanning, and the lack of fluorescence signaling indicated the absence of FITC-labeled gold-liposomes.

3.3. Blocking Gold-Liposome Uptake. Endocytosis is the process that cells use to take up nanovectors and other materials from the external environment, by encapsulating them in vesicles made from invaginations in the cell plasma membrane. It was of particular interest to investigate if the internalization of the gold-coupled liposomes could be hindered by a typical inhibitor of endocytosis. We used dynasore, a cell permeable, noncompetitive dynamin GTPase activity inhibitor, to block dynamin-dependent endocytosis of the liposomes (Figure 6). Our experiments indicated that the "gold-liposome" clusters barely attached to the external walls of cells treated $15 \mathrm{~min}$ with $80 \mu \mathrm{M}$ dynasore and then incubated with the "gold-liposomes" for $60 \mathrm{~min}$. In most of the cases, the "gold-liposomes" were easily removed from the cell membrane when probed with the AFM cantilever. The force loading exercised by the AFM probe, scanning to a velocity of $30 \mu \mathrm{m} / \mathrm{s}$, removed $\sim 90 \%$ of the liposomes. This indicated a poor binding efficiency with the plasma membrane and obstruction of the "gold-liposome" uptake after dynasore pretreatments.

\section{Conclusions}

Ninety-nanometer colloidal gold particles are a useful noninvasive labeling agent for visualization by AFM of liposome uptake by HCAECs. We were able to visualize the movement of the gold-coupled liposomes through the cell membrane before absorption. The time course of endocytosis was as follows: liposome attachment to the plasma membrane at 15$30 \mathrm{~min}$, internalization at 30-60 min, and liposome uptake completed at $120 \mathrm{~min}$. "Gold-liposome" clusters up to $\sim 3 \mu \mathrm{m}$ in diameter can be efficiently taken up by endocytosis regardless of their geometric structure.

The gold-coupled liposomes behaved as expected when exposed to an endocytosis inhibitor (dynasore) to block their internalization process. The gold nanoparticles did not hinder liposome uptake. We successfully established a potential method to track biomolecules in complex systems using $90 \mathrm{~nm}$ colloidal gold nanoparticles as a noninvasive contrast agent to improve AFM imaging.

\section{Acknowledgments}

The authors thank Dr. Russell Lebovitz of Marval Biosciences for his wise assistance during the development of this project and Dr. Maureen E. Goode, administrative director at the Center for Clinical and Translational Sciences, University of Texas Health Science Center at Houston for reviewing this paper. This work was supported by 


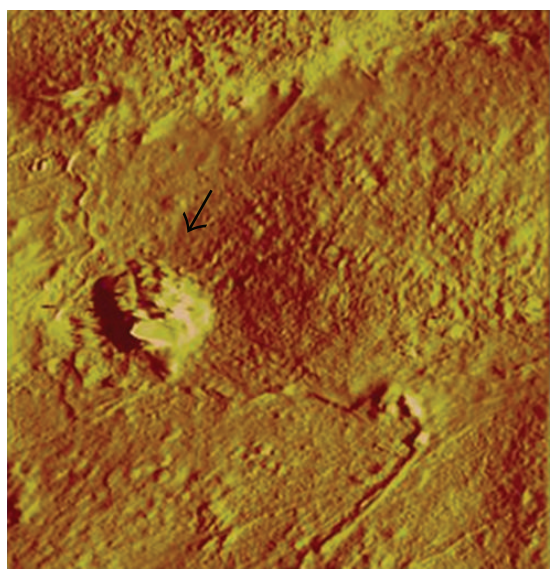

(a)

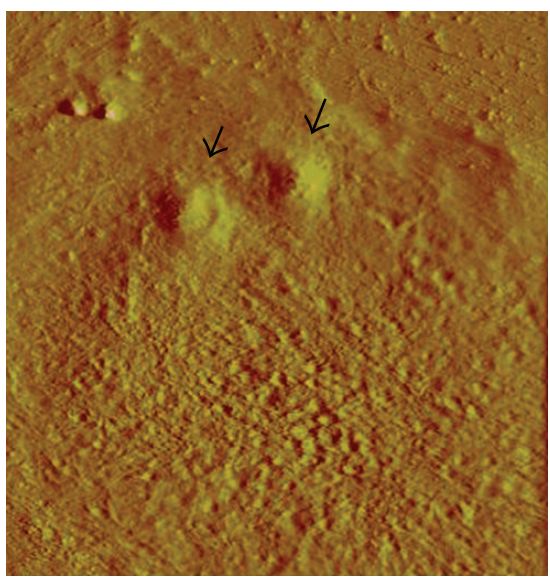

(c)

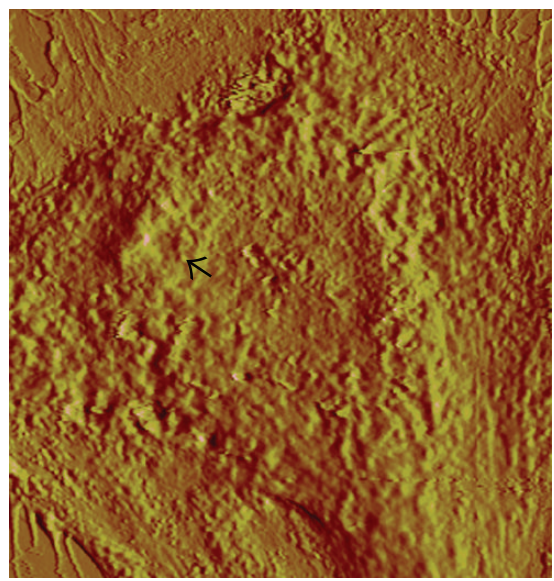

(e)

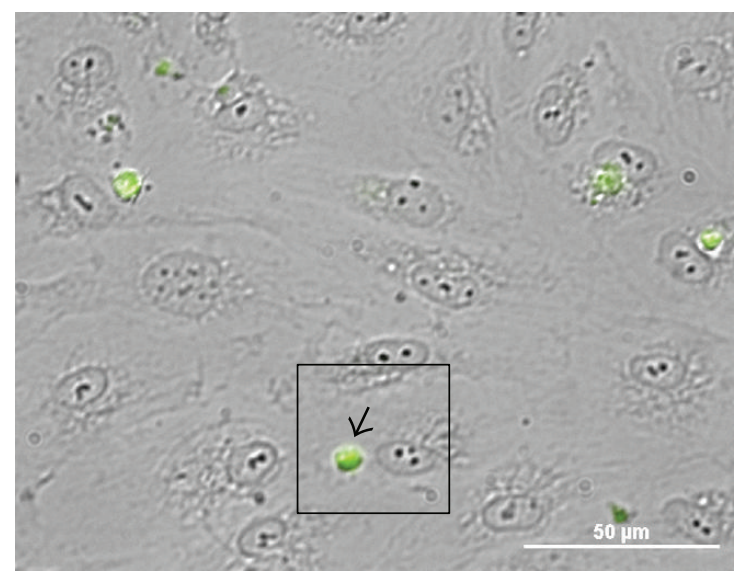

(b)

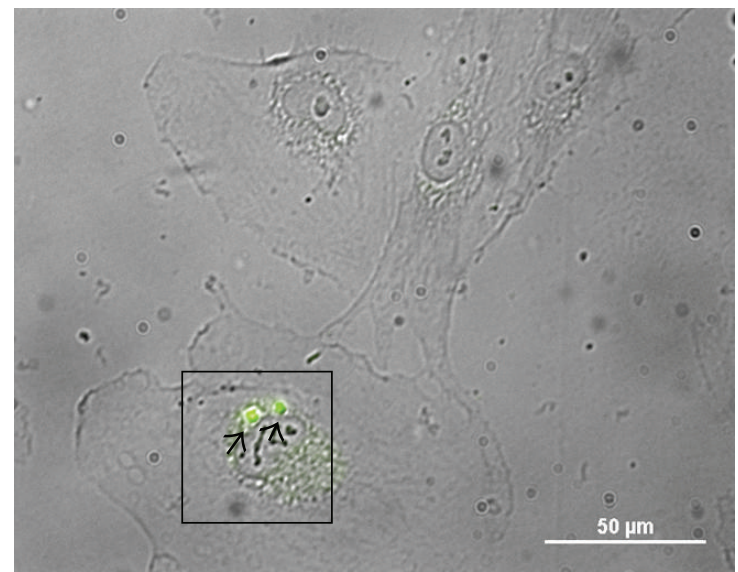

(d)

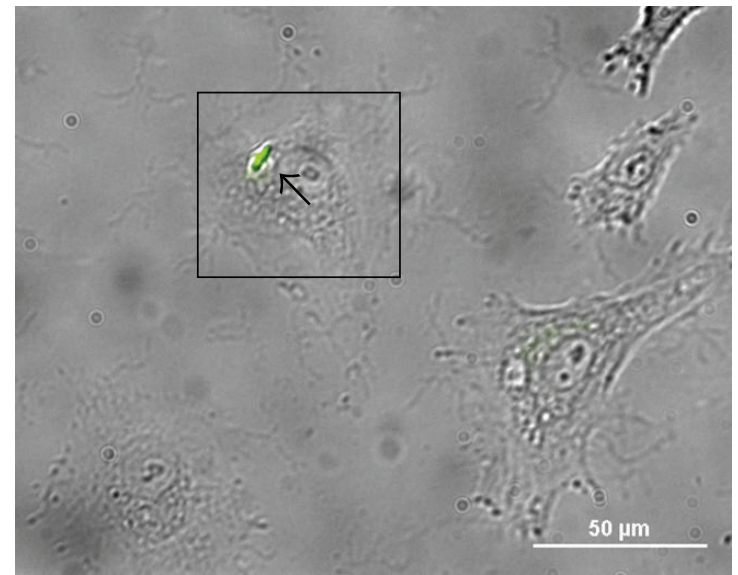

(f)

Figure 4: AFM images ((a), (c), and (e)) and corresponding fluorescence images ((b), (d), and (f)) of HCAECs incubated for 15 ((a) and (b)), 60 ((c) and (d)), and $120 \mathrm{~min}((\mathrm{e})$ and (f)) with FITC-labeled liposomes conjugated with $90 \mathrm{~nm}$ gold particles. The cells with positive signaling in the bright field images (squares in the right-hand panels) were selected for AFM scanning. (a) Typical AFM image of an HCAEC incubated for $15 \mathrm{~min}$, showing a gold liposome cluster attached to the cell membrane. (c) AFM image demonstrating the internalization process occurring in an HCAEC incubated for 60 min with "gold liposomes." (e) The AFM micrograph of an HCAEC incubated for 120 min corroborated that the "gold liposomes" internalized almost completely at this time point. Cells fixed in formalin $10 \%$ and scanned in contact mode in liquid (DNP-S fo $=12-24 \mathrm{kHz}, k=0.06 \mathrm{~N} / \mathrm{m}$ ). 


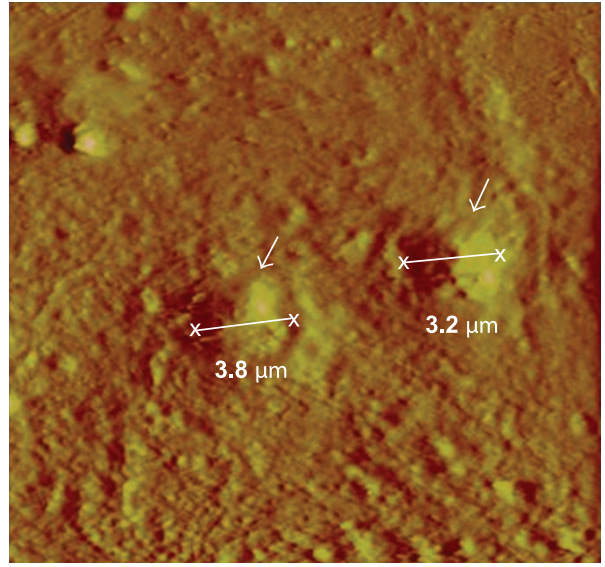

(a)

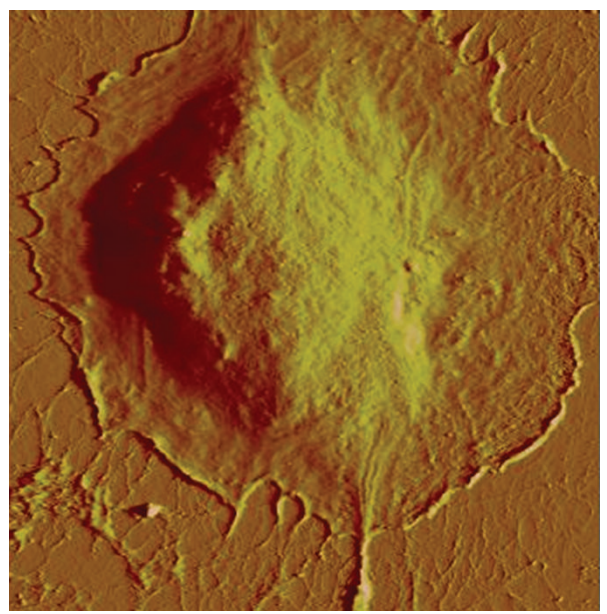

(c)

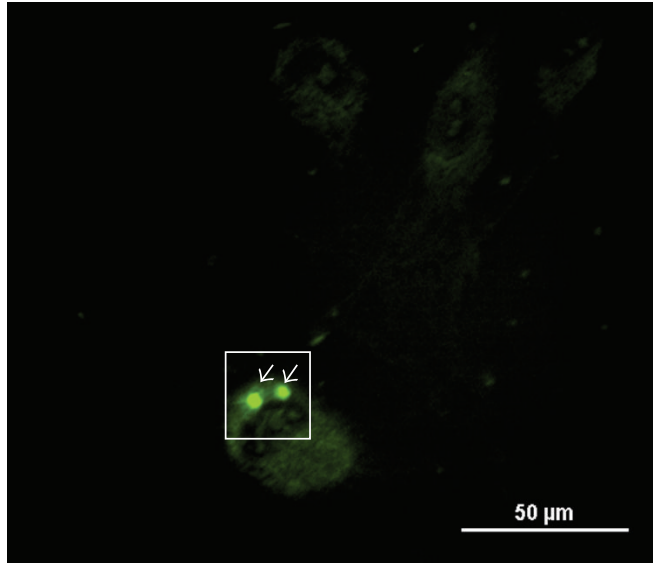

(b)

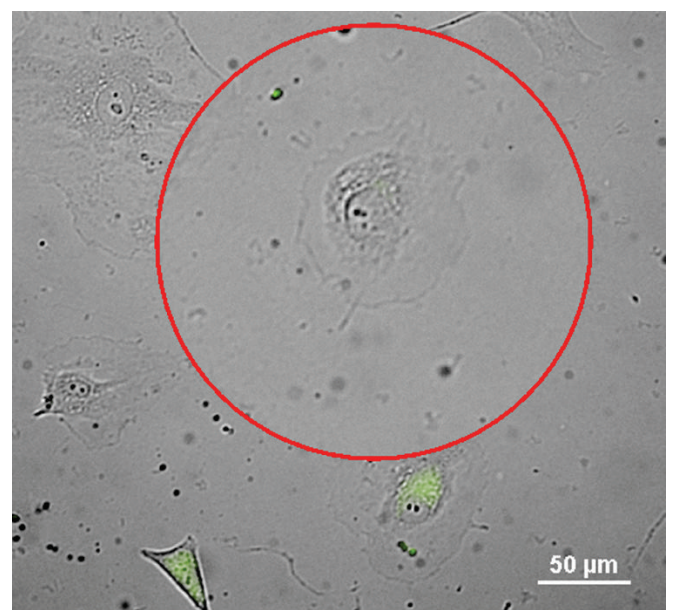

(d)

Figure 5: Liposome endocytosis occurring at 60 min of incubation (smaller scan area from Figure 4(c)). (a) AFM micrograph obtained at $25 \mu \mathrm{m}(x-y)$ illustrating the engulfment of the "gold liposomes" during cell membrane internalization. (b) The corresponding fluorescence image clearly shows the positive signaling emitted by the gold liposome clusters that were scanned. (c) AFM micrograph at $65 \mu \mathrm{m}(x-y)$ of an HCAEC negative control showing a smooth and even membrane surface after 60 min of incubation with FITC-labeled gold-liposomes. (d) The cells with no signalling, which indicated the absence of "gold-liposomes," were selected for scanning from the corresponding fluorescence images. Cells were fixed in formalin $10 \%$ and scanned in contact mode in liquid (DNP-S fo $=12-24 \mathrm{kHz}, k=0.06 \mathrm{~N} / \mathrm{m}$ ).

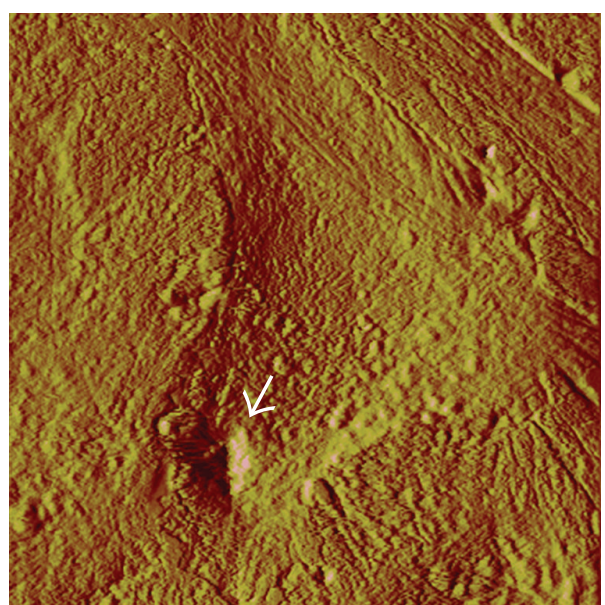

(a)

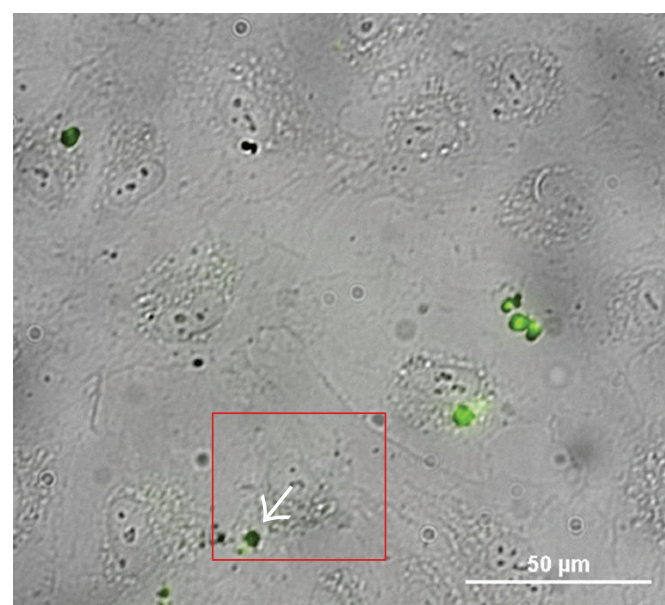

(b)

Figure 6: The internalization process of coupled liposomes was blocked when the cells were pretreated with $80 \mu \mathrm{M}$ dynasore for 15 min before 60 min of incubation with "gold-liposomes." The gold liposome clusters did not penetrate the cell membrane, remaining just attached to the external wall (arrow in (a)). The corresponding fluorescence image (b) shows positive signaling from the "gold liposomes" that were scanned. The cells were fixed in formalin $10 \%$ and imaged at $50 \mu \mathrm{m}^{2}$ in contact mode in liquid (DNP-S fo $=12-24 \mathrm{kHz}, k=0.06 \mathrm{~N} / \mathrm{m}$ ). 
DoD/TATCR Grants W81XWH-04-2-0035 (T5), W81XWH07-2-0031 (TexSHIELD), and NIH Clinical and Translational Science Award UL1 RR024148.

\section{References}

[1] S. Anabousi, M. Laue, C. M. Lehr, U. Bakowsky, and C. Ehrhardt, "Assessing transferrin modification of liposomes by atomic force microscopy and transmission electron microscopy," European Journal of Pharmaceutics and Biopharmaceutics, vol. 60, no. 2, pp. 295-303, 2005.

[2] G. Blume and G. Cevc, "Liposomes for the sustained drug release in vivo," Biochimica et Biophysica Acta, vol. 1029, no. 1, pp. 91-97, 1990.

[3] A. L. Klibanov, K. Maruyama, V. P. Torchilin, and L. Huang, "Amphipathic polyethyleneglycols effectively prolong the circulation time of liposomes," FEBS Letters, vol. 268, no. 1, pp. 235237, 1990.

[4] G. Bendas, A. Krause, U. Bakowsky, J. Vogel, and U. Rothe, "Targetability of novel immunoliposomes prepared by a new antibody conjugation technique," International Journal of Pharmaceutics, vol. 181, no. 1, pp. 79-93, 1999.

[5] A. Udhrain, K. M. Skubitz, and D. W. Northfelt, "Pegylated liposomal doxorubicin in the treatment of AIDS-related Kaposi's sarcoma," Journal of Nanomedicine, vol. 2, no. 3, pp. 345-352, 2007.

[6] F. Luderer, K. Sternberg, H. W. Rohm et al., "Suitability of nanoparticles for stent application," in Proceedings of the 4th European Conference of the International Federation for Medical and Biological Engineering (ECIFMBE '08), vol. 22, pp. 23392342, November 2008.

[7] J. F. Hainfeld and R. D. Powell, "New frontiers in gold labeling," Journal of Histochemistry and Cytochemistry, vol. 48, no. 4, pp. 471-480, 2000.

[8] J. E. Beesley, "Colloidal gold for microbiological immunocytochemistry," in Colloidal Gold: Principles, Methods and Applications, M. A. Hayat, Ed., vol. 1, pp. 421-425, Academic Press, New York, NY, USA, 1989.

[9] D. Danila, R. Partha, D. B. Elrod, M. Lackey, S. W. Casscells, and J. L. Conyers, "Antibody-labeled liposomes for CT imaging of atherosclerotic plaques: in vitro investigation of an anti-ICAM antibody-labeled liposome containing iohexol for molecular imaging of atherosclerotic plaques via computed tomography," Texas Heart Institute Journal, vol. 36, no. 5, pp. 393-403, 2009.

[10] K. Laginha, D. Mumbengegwi, and T. Allen, "Liposomes targeted via two different antibodies: assay, B-cell binding and cytotoxicity," Biochimica et Biophysica Acta, vol. 1711, no. 1, pp. 25-32, 2005.

[11] C. B. Hansen, G. Y. Kao, E. H. Moase, S. Zalipsky, and T. M. Allen, "Attachment of antibodies to sterically stabilized liposomes: evaluation, comparison and optimization of coupling procedures," Biochimica et Biophysica Acta, vol. 1239, no. 2, pp. 133-144, 1995.

[12] M. Sartor, University of California San Diego, Index of neurophysics courses, (December, 2011), http://physics.ucsd.edu/ neurophysics/courses/physics_173_273/dynamic_light_scattering_03.pdf.

[13] K. Maruyama, T. Takizawa, N. Takahashi, T. Tagawa, K. Nagaike, and M. Iwatsuru, "Targeting efficiency of PEGimmunoliposome-conjugated antibodies at PEG terminals," Advanced Drug Delivery Reviews, vol. 24, no. 2-3, pp. 235-242, 1997.
[14] S. Ramachandran, A. P. Quist, S. Kumar, and R. Lal, "Cisplatin nanoliposomes for cancer therapy: AFM and fluorescence imaging of cisplatin encapsulation, stability, cellular uptake, and toxicity," Langmuir, vol. 22, no. 19, pp. 8156-8162, 2006.

[15] S. Sever, "Dynamin and endocytosis," Current Opinion in Cell Biology, vol. 14, no. 4, pp. 463-467, 2002.

[16] S. J. McClure and P. J. Robinson, "Dynamin, endocytosis and intracellular signalling," Molecular Membrane Biology, vol. 13, no. 4, pp. 189-215, 1996.

[17] J. E. Hinshaw, "Dynamin and its role in membrane fission," Annual Review of Cell and Developmental Biology, vol. 16, pp. 483-519, 2000.

[18] K. N. Fish, S. L. Schmid, and H. Damke, "Evidence that dynamin-2 functions as a signal-transducing GTPase," Journal of Cell Biology, vol. 150, no. 1, pp. 145-154, 2000.

[19] A. J. Newton, T. Kirchhausen, and V. M. Murthy, "Inhibition of dynamin completely blocks compensatory synaptic vesicle endocytosis," Proceedings of the National Academy of Sciences of the United States of America, vol. 103, no. 47, pp. 17955-17960, 2006.

[20] E. Mastrobattista, G. Storm, L. Van Bloois et al., "Cellular uptake of liposomes targeted to intercellular adhesion molecule-1 (ICAM-1) on bronchial epithelial cells," Biochimica et Biophysica Acta, vol. 1419, no. 2, pp. 353-363, 1999.

[21] V. Hengst, C. Oussoren, T. Kissel, and G. Storm, "Bone targeting potential of bisphosphonate-targeted liposomes. Preparation, characterization and hydroxyapatite binding in vitro," International Journal of Pharmaceutics, vol. 331, no. 2, pp. 224-227, 2007.

[22] S. A. Jackson and R. M. Thomas, Cross-Sectional Imaging Made Easy, Churchill Livingstone, New York, NY, USA, 2004.

[23] G. Puu, E. Artursson, I. Gustafson, M. Lundström, and J. Jass, "Distribution and stability of membrane proteins in lipid membranes on solid supports," Biosensors and Bioelectronics, vol. 15, no. 1-2, pp. 31-41, 2000.

[24] I. Ruspantini, M. Diociaiuti, R. Ippoliti et al., "Immunogold localisation of P-glycoprotein in supported lipid bilayers by transmission electron microscopy and atomic force microscopy," Histochemical Journal, vol. 33, no. 5, pp. 305-309, 2001. 

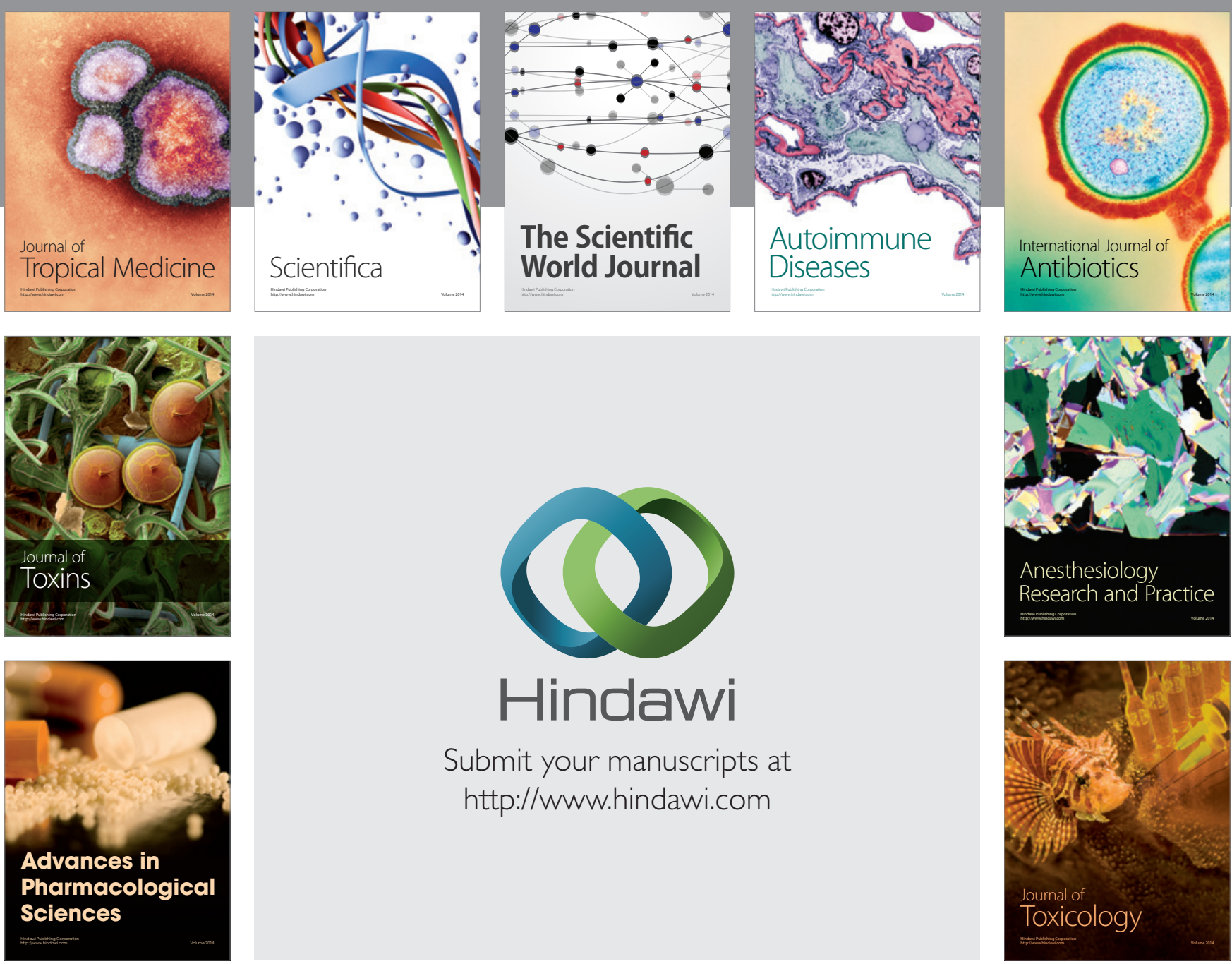

\section{Hindawi}

Submit your manuscripts at

http://www.hindawi.com
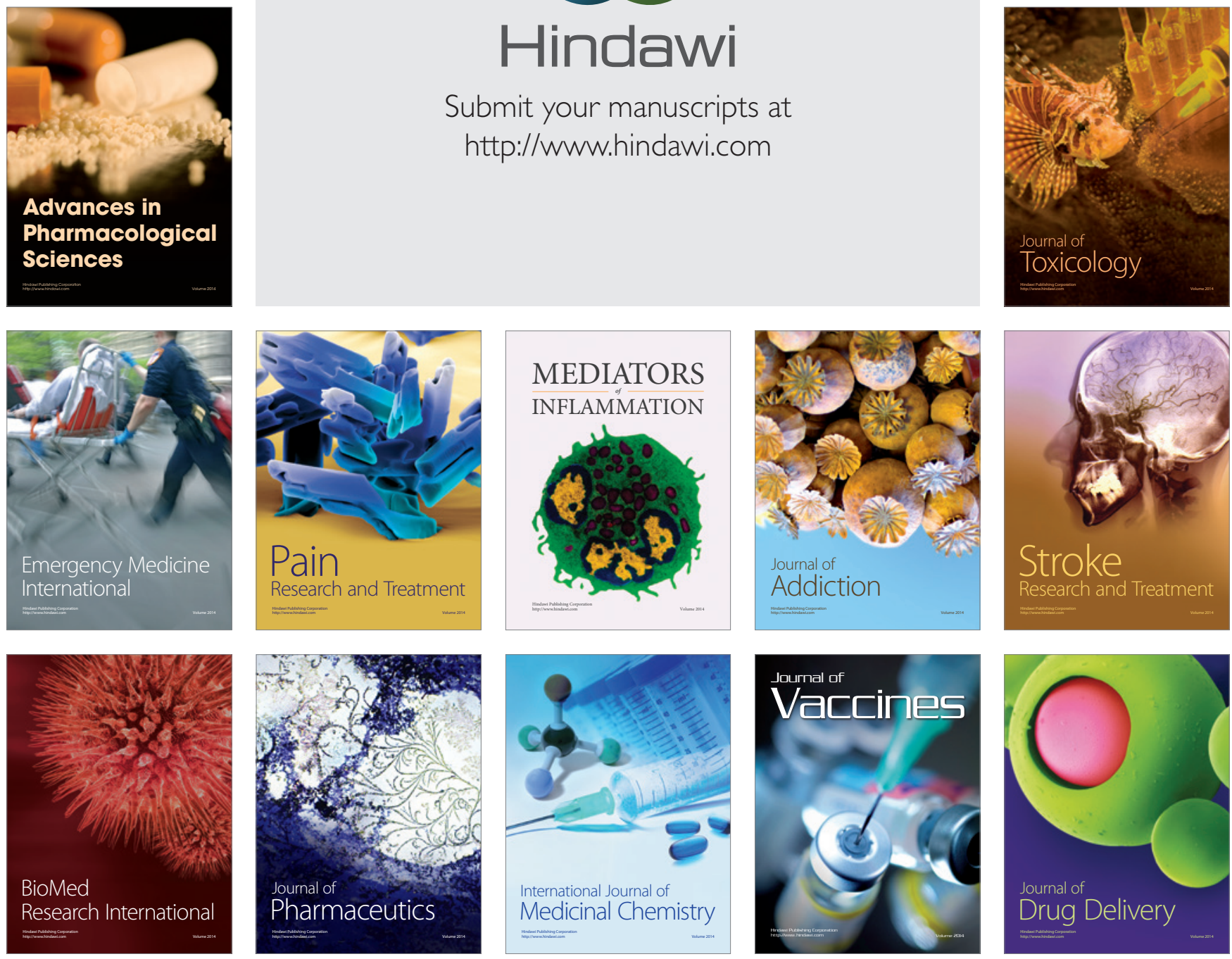